\title{
Genetic variants in PTPRD and risk of gestational diabetes mellitus
}

\author{
Ting Chen ${ }^{1,2,3, *}$, Juan $X u^{3,4, *}$, Guangquan Liu ${ }^{3}$, Heng Liu ${ }^{3}$, Minjian Chen ${ }^{1,2}$, Yufeng \\ Qin $^{5}$, Wei Wu ${ }^{1,2}$, Yankai Xiaa ${ }^{1,2}$, Chenbo $\mathrm{Ji}^{3,6}$, Xirong Guo ${ }^{3,6}$, Juan Wen ${ }^{3,6}$, Xinru Wang ${ }^{1,2}$ \\ ${ }^{1}$ State Key Laboratory of Reproductive Medicine, Institute of Toxicology, Nanjing Medical University, Nanjing 211166, China \\ ${ }^{2}$ Key Laboratory of Modern Toxicology of Ministry of Education, School of Public Health, Nanjing Medical University, Nanjing \\ 211166, China \\ ${ }^{3}$ Nanjing Maternity and Child Health Care Institute, Nanjing Maternity and Child Health Care Hospital Affiliated to Nanjing \\ Medical University, Nanjing 210004, China \\ ${ }^{4}$ Department of Obstetrics and Gynecology, Nanjing Maternity and Child Health Care Hospital Affiliated to Nanjing Medical \\ University, Nanjing 210004, China \\ ${ }^{5}$ Epigenetics \& Stem Cell Biology Laboratory, National Institute of Environmental Health Sciences, Research Triangle Park, \\ NC 27709, USA \\ ${ }^{6}$ Department of Children Health Care, Nanjing Maternity and Child Health Care Hospital Affiliated to Nanjing Medical \\ University, Nanjing 210004, China \\ *These authors have contributed equally to this work \\ Correspondence to: Juan Wen, email: wenj2010@gmail.com \\ Xinru Wang, email: xrwang@njmu.edu.cn
}

Keywords: PTPRD, polymorphism, gestational diabetes mellitus, susceptibility

Received: August 05, 2016

Accepted: September 29, 2016

Published: October 12, 2016

\section{ABSTRACT}

Genome-wide association studies (GWASs) showed that two single nucleotide polymorphisms (SNPs) (rs17584499 and rs649891) in the protein tyrosine phosphatase receptor type D (PTPRD) were associated with type 2 diabetes (T2D). We sought to determine the influence of the PTPRD variants on the gestational diabetes mellitus (GDM) risk. In this research, two SNPs in PTPRD reported in T2D GWASs and six PTPRD expression-related SNPs were genotyped in 964 GDM cases and 1,021 controls using the Sequenom platform. Logistic regression analyses in additive models showed consistently significant associations of PTPRD rs10511544 A>C, rs10756026 T>A and rs10809070 C>G with a decreased risk of GDM [adjusted OR (95\% CI) $=0.83(0.72-0.97)$ for rs 10511544 ; adjusted $O R(95 \% \mathrm{CI})=0.81(0.70-0.94)$ for rs10756026; adjusted OR $(95 \% \mathrm{CI})=0.78(0.65-0.92)$ for rs10809070]. Furthermore, the risk of GDM was significantly decreased with an increasing number of variant alleles of the three SNPs in a dose-dependent manner $\left(P_{\text {trend }}=\mathbf{0 . 0 0 8}\right)$. Moreover, the haplotype containing variant alleles of the three SNPs were significantly associated with a decreased risk of GDM [adjusted OR $(95 \% \mathrm{CI})=0.77(0.64-0.92), P=0.005$ ], when compared with the most frequent haplotype. However, there were no significant associations for the SNPs reported in the T2D GWASs. Altogether, these findings indicate that the variants of rs10511544, rs10756026 and rs10809070 in PTPRD may contribute to a decreased susceptibility to GDM. Further validation in different ethnic backgrounds and biological function analyses are needed.

\section{INTRODUCTION}

Gestational diabetes mellitus (GDM) is defined as varying degrees of glucose intolerance with an onset or first recognition during pregnancy [1]. The incidence of GDM has steadily risen in the last few decades and affects $1 \%-14 \%$ of all pregnancies [2]. Both GDM patients and their children have an increased risk of developing type 2 
diabetes (T2D) and metabolic syndrome later in life [3]. Human and animal studies suggested that T2D and GDM may have some common pathological changes, including insulin resistance, $\beta$-cell dysfunction and defects in insulin action [4], and may share predisposing factors. As there is evidence for a higher prevalence of T2D in mothers of GDM patients and the clustering of impaired glucose tolerance and T2D in families with GDM patients [5], GDM may have similar genetic background as T2D.

In the past few decades, genetic studies, including genome-wide association studies (GWASs) and candidate gene strategies, have identified many susceptibility genes involved in the increased risk of T2D and GDM [6, 7]. There has been progress in finding the genetic risk factors for GDM in relation to T2D. Some of the genetic variants that were proven to be genetic risk factors for T2D are also significantly associated with GDM [7, 8]. In 2010, Tsai et al. performed a two-stage GWAS (995 T2D patients and 894 controls for the first genome scan) and identified rs 17584499 in the protein tyrosine phosphatase receptor type $\mathrm{D}(P T P R D)$ gene for T2D in a Han Chinese population [9]. Then, Below et al. performed another GWAS with 837 T2D cases and 436 normoglycaemic controls for a GWA scan, followed by a meta-analysis, and found such an association with another SNP, rs649891, in $P T P R D$ in Mexican-Americans [10]. In a replication study, the PTPRD genetic variant was suggested to be associated with progression to diabetes in Han Chinese, most likely through increased insulin resistance [11]. Recently, Chen et al. found that the levels of PTPRD were significantly lower in T2D patients, and this protein was involved in the insulin signalling pathway [12]. However, few studies have investigated the associations of the genetic variants of PTPRD with the risk of GDM.

In a Korean population, some of the T2D-associated genetic variants discovered in GWASs were suggested to also be associated with GDM [7]. Therefore, in this study, we hypothesized that genetic variants in PTPRD might also contribute to the risk of GDM. To test the hypothesis, we selected 8 single nucleotide polymorphisms (SNPs) in PTPRD and performed a case-control study, including 964 GDM cases and 1,021 controls, to test the associations of the SNPs with the risk of GDM.

\section{RESULTS}

The selected characteristics of the 964 GDM patients and 1,021 controls are shown in Table 1. As expected, there were similar distributions of age and pre-pregnancy body mass index (BMI) between the two groups $(P=$ 0.094 and 0.685 ). However, there were more multiparaes, women with abnormal pregnancy histories and women with family histories of diabetes among the GDM cases than among the controls ( $P<0.05$ for all comparisons).
Logistic regression analyses in different genetic models were used to examine the associations between the 8 studied SNPs and GDM susceptibility. Among the controls, the genotype frequencies of the 8 SNPs were all in Hardy-Weinberg equilibrium $(P>0.05$ for all SNPs). We did not validate the results of the PTPRD variations (rs17584499 and rs649891) reported in the published GWASs for T2D, but we observed that PTPRD rs $10511544 \mathrm{~A}>\mathrm{C}$, rs10756026 $\mathrm{T}>\mathrm{A}$ and $\mathrm{rs} 10809070 \mathrm{C}>\mathrm{G}$ were significantly associated with a decreased risk of GDM using the additive model [adjusted OR $(95 \% \mathrm{CI})=$ 0.83 (0.72-0.97) for rs 10511544; adjusted OR (95\% CI) $=0.81(0.70-0.94)$ for rs 10756026 ; adjusted OR $(95 \%$ $\mathrm{CI})=0.78$ (0.65-0.92) for rs10809070] (Table 2-3). Then, conditional logistic regression analyses were used to test the independence of the three significant SNPs. The effects of rs10511544 and rs10809070 on GDM occurrence were weakened after being conditioned on the other two SNPs. However, the effect of rs 10756026 on GDM occurrence persisted after being conditioned on the other two SNPs [adjusted OR $(95 \% \mathrm{CI})=0.71(0.51-0.99)]$.

Then, we evaluated the combined effects on GDM occurrence by adding the number of variant alleles of the three significant SNPs (rs10511544-C, rs10756026-A and rs10809070-G). The "0" allele means subjects with wide-type homozygotes of the three SNPs; "1-6" alleles means carrying one to six variant alleles of the three SNPs. The results showed that the GDM risk was significantly decreased with an increasing number of variant alleles of the three SNPs in a dose-dependent manner $\left(P_{\text {trend }}=\right.$ 0.008 ) (Table 4). When compared with the subjects with wide-type homozygotes of the three SNPs, subjects carrying four to six variant alleles had a $46 \%$ decrease in GDM risk (95\% CI $=0.37-0.81)$ (Table 4). The combined effects of the three SNPs on GDM occurrence were also evaluated by stratifying by age, pre-pregnancy BMI, parity, abnormal pregnancy history and family history of diabetes. No obvious evidence of heterogeneity associations for the combined effects of the three SNPs on GDM risk was observed (Supplementary Table S1). We also conducted stratified analyses on rs 10511544 , rs10756026 and rs10809070 with GDM susceptibility, respectively (Supplementary Table S2-S4). There was also no heterogeneity between the similar association strengths of the subgroups $(P>0.05)$.

The linkage disequilibrium (LD) information of the three SNPs, calculated from genotyping data of the control group, is shown in Supplementary Table S5. We conducted haplotype analyses to evaluate the effect of the haplotype containing variant alleles of the three SNPs on GDM occurrence (Table 5). When compared with the most frequent ATC haplotype, the haplotype containing variant alleles of the three SNPs (CAG) was significantly associated with a decreased risk of GDM [adjusted (95\% 
Table 1: Demographic and selected variables in GDM cases and controls

\begin{tabular}{|c|c|c|c|}
\hline Variables & GDM cases $(n=964) N(\%)$ & Controls $(n=1021) N(\%)$ & $P$ \\
\hline Age, year $($ mean $\pm S D)$ & $30.6 \pm 3.7$ & $30.3 \pm 3.6$ & 0.094 \\
\hline $\begin{array}{l}\text { Pre-pregnancy BMI, } \mathrm{kg} / \mathrm{m}^{2} \\
(\text { mean } \pm \mathrm{SD})\end{array}$ & $22.1 \pm 2.9$ & $22.0 \pm 2.8$ & 0.685 \\
\hline Parity & & & $<0.001$ \\
\hline Nulliparae & $827(85.8)$ & $953(93.3)$ & \\
\hline Multiparae & $137(14.2)$ & $68(6.7)$ & \\
\hline Abnormal pregnancy history & & & $<0.001$ \\
\hline No & $847(87.9)$ & $981(96.1)$ & \\
\hline Yes & $117(12.1)$ & $40(3.9)$ & \\
\hline Family history of diabetes & & & 0.023 \\
\hline No & $791(82.1)$ & $876(85.8)$ & \\
\hline Yes & $173(17.9)$ & $145(14.2)$ & \\
\hline
\end{tabular}

Abbreviation: GDM, gestational diabetes mellitus; SD, standard deviation; BMI, body mass index.

Table 2: Genotyping results in GDM cases and controls

\begin{tabular}{|c|c|c|c|c|c|c|}
\hline \multirow[b]{2}{*}{ SNP } & \multirow[b]{2}{*}{ Base chang } & \multirow[b]{2}{*}{$\mathbf{M A F}^{\mathbf{b}}$} & \multirow[b]{2}{*}{ Reported MAF ${ }^{\mathrm{c}}$} & \multicolumn{3}{|c|}{$P$ value } \\
\hline & & & & $\begin{array}{c}\text { Dominant } \\
\text { model }\end{array}$ & $\begin{array}{c}\text { Recessive } \\
\text { model }\end{array}$ & Additive model \\
\hline rs17584499 & $\mathrm{C}>\mathrm{T}$ & 0.090 & 0.087 & 0.331 & 0.076 & 0.190 \\
\hline rs649891 & $\mathrm{C}>\mathrm{T}$ & 0.217 & 0.223 & 0.418 & 0.555 & 0.654 \\
\hline rs10511544 & $\mathrm{A}>\mathrm{C}$ & 0.267 & 0.267 & 0.116 & 0.003 & 0.015 \\
\hline rs 10756026 & $\mathrm{~T}>\mathrm{A}$ & 0.253 & 0.262 & 0.041 & 0.003 & 0.005 \\
\hline rs10809070 & $\mathrm{C}>\mathrm{G}$ & 0.180 & 0.175 & 0.047 & $<0.001$ & 0.004 \\
\hline rs 12345848 & $\mathrm{G}>\mathrm{A}$ & 0.177 & 0.180 & 0.546 & 0.134 & 0.318 \\
\hline rs1323500 & $\mathrm{T}>\mathrm{G}$ & 0.434 & 0.437 & 0.370 & 0.218 & 0.200 \\
\hline rs628731 & $\mathrm{A}>\mathrm{G}$ & 0.250 & 0.257 & 0.243 & 0.013 & 0.058 \\
\hline
\end{tabular}

Notes: ${ }^{a}$ Major $>$ minor allele; ${ }^{b}$ MAF in 1021 controls; ${ }^{c}$ Reported MAF in Han Chinese from 1,000 genomes.

Abbreviations: GDM, gestational diabetes mellitus; SNP, single nucleotide polymorphism; MAF, minor allele frequency.

$\mathrm{CI})=0.77(0.64-0.92), P=0.005)]$, which was consistent with the single SNP analysis.

\section{DISCUSSION}

As far as we know, this is the first study that has provided evidence that common SNPs in PTPRD might be associated with GDM susceptibility. We found that PTPRD rs10511544 A $>$ C, rs $10756026 \mathrm{~T}>\mathrm{A}$ and rs $10809070 \mathrm{C}>\mathrm{G}$ were significantly associated with a decreased risk of GDM. Additionally, GDM risk significantly decreased with an increasing number of variant alleles of the three SNPs in a dose-dependent manner.
PTPRD belongs to the R2A subfamily of protein tyrosine phosphatases (PTPs), which have been implicated in cancer, neural development, and diabetes [13]. PTPRD is expressed widely, including the brain, the pancreas and skeletal muscle. Multiple mRNA isoforms are expressed by alternative transcription start sites (TSSs) and/or alternative splicing in a tissue- and developmentalspecific manner [14]. PTPRD is one of the most frequently inactivated genes across human cancers [15]. In gliomas, loss of PTPRD could cause aberrant activation of STAT3 and is closely associated with glioma progression [16]. PTPRD-deficient mice exhibit neonatal mortality, impaired learning and memory, posture and motor defects, 
Table 3: Association between 3 significant SNPs and GDM susceptibility

\begin{tabular}{|c|c|c|c|c|c|c|}
\hline Genotype & $\begin{array}{c}\text { GDM cases } \\
(n=964) \mathbf{N}(\%)\end{array}$ & $\begin{array}{l}\text { Controls }(\mathrm{n}= \\
1021) \text { N }(\%)\end{array}$ & OR $(95 \% C I)$ & $P$ & OR $(95 \% C I)^{a}$ & $P^{a}$ \\
\hline \multicolumn{7}{|l|}{ rs 10511544} \\
\hline AA & $562(58.7)$ & $559(54.8)$ & 1.00 & & 1.00 & \\
\hline $\mathrm{CA}$ & $348(36.3)$ & $378(37.0)$ & $0.92(0.76-1.10)$ & 0.356 & $0.93(0.77-1.13)$ & 0.472 \\
\hline $\mathrm{CC}$ & $48(5.0)$ & $84(8.2)$ & $0.57(0.39-0.83)$ & 0.003 & $0.56(0.38-0.82)$ & 0.003 \\
\hline Additive & & & $0.83(0.72-0.96)$ & 0.011 & $0.83(0.72-0.97)$ & 0.015 \\
\hline \multicolumn{7}{|l|}{ rs 10756026} \\
\hline $\mathrm{TT}$ & $591(61.4)$ & $573(56.6)$ & 1.00 & & 1.00 & \\
\hline AT & $332(34.5)$ & $366(36.2)$ & $0.88(0.73-1.06)$ & 0.180 & $0.89(0.73-1.07)$ & 0.217 \\
\hline $\mathrm{AA}$ & $40(4.2)$ & $73(7.2)$ & $0.53(0.36-0.79)$ & 0.002 & $0.53(0.35-0.80)$ & 0.002 \\
\hline Additive & & & $0.81(0.70-0.93)$ & 0.004 & $0.81(0.70-0.94)$ & 0.005 \\
\hline \multicolumn{7}{|l|}{ rs 10809070} \\
\hline $\mathrm{CC}$ & $691(72.9)$ & $700(68.6)$ & 1.00 & & 1.00 & \\
\hline $\mathrm{GC}$ & $241(25.4)$ & $273(26.8)$ & $0.89(0.73-1.10)$ & 0.280 & $0.90(0.73-1.11)$ & 0.313 \\
\hline GG & $16(1.7)$ & $47(4.6)$ & $0.35(0.19-0.61)$ & $<0.001$ & $0.34(0.19-0.62)$ & $<0.001$ \\
\hline Additive & & & $0.77(0.65-0.92)$ & 0.003 & $0.78(0.65-0.92)$ & 0.004 \\
\hline
\end{tabular}

Note: ${ }^{a}$ Logistic regression analyses adjusted for age, pre-pregnancy BMI, parity, abnormal pregnancy history and family history of diabetes. Abbreviations: GDM, gestational diabetes mellitus; SNP, single nucleotide polymorphism.

Table 4: Cumulative effects of variant alleles on GDM susceptibility

\begin{tabular}{|c|c|c|c|c|c|c|}
\hline Variables & $\begin{array}{l}\text { GDM cases }(n= \\
964) \text { N (\%) }\end{array}$ & $\begin{array}{c}\text { Controls }(n=1021) \\
\text { N }(\%)\end{array}$ & OR $(95 \% C I)$ & $P$ & OR $(95 \% C I)^{a}$ & $P a$ \\
\hline \multicolumn{7}{|c|}{ Combined effects of rs10511544-C, rs10756026-A and rs10809070-G } \\
\hline 0 & $546(57.72)$ & $538(53.21)$ & 1.00 & & 1.00 & \\
\hline $1-3$ & $356(37.63)$ & 393 (38.87) & $0.89(0.74-1.08)$ & 0.232 & $0.90(0.75-1.09)$ & 0.290 \\
\hline $4-6$ & $44(4.65)$ & $80(7.91)$ & $0.54(0.37-0.80)$ & 0.002 & $0.54(0.37-0.81)$ & 0.002 \\
\hline Trend & & & $P^{\mathrm{b}}=0.005$ & & $P^{\mathrm{b}}=0.008$ & \\
\hline
\end{tabular}

Note: ${ }^{a}$ Logistic regression analyses adjusted for age, pre-pregnancy BMI, parity, abnormal pregnancy history and family history of diabetes. ${ }^{\mathrm{b}} P$ value of Cochran-Armitage's trend test. Abbreviation: GDM, gestational diabetes mellitus.

Table 5: Results of haplotype association analyses

\begin{tabular}{lcccc}
\hline Haplotype & GDM N (\%) & Controls N (\%) & OR (95\%CI) & P \\
\hline ATC & $1464(75.9)$ & $1455(71.3)$ & 00 & 0.005 \\
CAG & $249(12.9)$ & $329(16.1)$ & $0.77(0.64-0.92)$ & 0.661 \\
CAC & $148(7.7)$ & $163(8.0)$ & $0.95(0.75-1.20)$ & 0.025 \\
Others $^{\text {a }}$ & $67(3.5)$ & $95(4.7)$ & $0.69(0.50-0.96)$ & \\
\hline
\end{tabular}

Note: Logistic regression analyses adjusted for age, pre-pregnancy BMI, parity, abnormal pregnancy history and family history of diabetes. SNPs order: rs16847024, rs17429130 and rs4917356. ${ }^{\text {a }}$ Haplotypes with a frequency less than 5\% in all three groups were combined as others. Abbreviation: GDM, gestational diabetes mellitus. 
and early growth retardation [17]. The R2A PTP subfamily includes PTPRD, PTPRS and LAR. These members are structurally very similar [14]. The overexpression of LAR in mouse skeletal muscle could cause whole-body insulin resistance [18]. The PTPRS- and LAR-deficient mice were demonstrated to have altered insulin sensitivity and glucose homeostasis [19]. Therefore, PTPRD may also play a role in the pathogenesis of T2D and GDM, and should be further characterized.

The SNPs rs $10511544 \mathrm{~A}>\mathrm{C}$, rs $10756026 \mathrm{~T}>\mathrm{A}$ and rs $10809070 \mathrm{C}>\mathrm{G}$ were located in the intron of PTPRD. Interestingly, the variant alleles of the three SNPs were associated with higher expression of PTPRD, according to the GTEx Portal (http://www.gtexportal.org) database, and PTPRD levels were reported to be lower in T2D patients [12], supporting the protective effect of the variant alleles of the three SNPs on GDM occurrence. Although this evidence for the three SNPs seems to be biologically plausible, lack of evidence for the functionality that links the three SNPs and PTPRD and then links the latter to GDM occurrence was one of the drawbacks of this study. Therefore, further functional analysis of the regions including the three SNPs is needed.

Our study had a number of strengths. First, our GDM cases and controls came from a systematic screening of pregnancy complications in a populationbased, large study performed in Nanjing, and the two groups were well matched based on age and prepregnancy BMI, which may have reduced a potential selection bias. Moreover, the relatively large sample size in this study provided enough statistical power. However, some reported risk factors for GDM, such as poor diet, low physical activity, and polycystic ovarian syndrome $[20,21]$, were not considered for adjustment in this study. Our study was underpowered to detect associations of some of the SNPs with GDM, especially for the two SNPs reported in the T2D GWAS (rs17584499 and rs649891), probably because of their lower frequencies, which may have resulted in some associations being overlooked. The heterogeneity among populations and diseases (T2D and GDM) may also contribute to the negative results. Therefore, the results should be treated with caution, and validation is warranted.

Altogether, our study suggested that PTPRD loci are candidate susceptibility regions that have some marker SNPs for GDM in Han Chinese. Further studies performed in diverse populations with functional assays are needed to validate and extend our findings.

\section{MATERIALS AND METHODS}

\section{Study subjects}

The study was approved by the institutional review board of Nanjing Maternity and Child Health Care Institute (Nanjing, China), and the methods were carried out in accordance with the approved guidelines. The case-control study was conducted on the basis of a study population of more than 80,000 women who had pregnancy complications screening between March 2012 and February 2015 at Nanjing Maternity and Child Health Care Hospital (Nanjing, China). The GDM cases and controls were randomly selected from the maternal screening population using a computerized random number function. All participants were offered a glucose challenge test (GCT) between weeks 24 and 28 of gestation. The GDM cases were defined as pregnant women with fasting glucose concentration $\geq 5.5 \mathrm{mmol} / \mathrm{L}$ or 2-h plasma glucose concentration $\geq 8.0 \mathrm{mmol} / \mathrm{L}$ [22] Women diagnosed with diabetes before pregnancy were excluded from this study. Pregnant women without diabetes were included as controls. The selected controls were matched to the GDM patients based on age and pre-pregnancy BMI and declared to have no previous metabolic diseases. As a result, $964 \mathrm{GDM}$ cases and 1,021 controls consented to participate in the study. After written informed consent was obtained, each participant was scheduled for an interview using a structured questionnaire to collect demographic information and potential risk factors, such as age, pre-pregnancy height and weight, parity, abnormal pregnancy history and family history of diabetes. All cases and controls were unrelated ethnic Han Chinese.

\section{SNPs selection and genotyping}

Two SNPs in PTPRD (rs17584499 and rs649891) reported in T2D GWAS and common PTPRD expression quantitative trait loci (eQTLs) SNPs were included for analysis. The selected eQTLs SNPs had minor allele frequency (MAF) larger than 0.05 in Han Chinese and were associated with $P T P R D$ expression according to the GTEx Portal Database (last search date: December 2015). If SNPs were in high linkage disequilibrium $\left(\mathrm{r}^{2}>0.8\right)$, we genotyped only one SNP. As a result, the two GWASreported SNPs in PTPRD (rs17584499 and rs649891) and six PTPRD eQTLs SNPs (rs10511544, rs10756026, rs10809070, rs12345848, rs1323500 and rs628731) were selected for genotyping (Table 2).

Genomic DNA was extracted from leukocyte pellets by traditional proteinase $\mathrm{K}$ digestion, followed by phenolchloroform extraction and ethanol precipitation. All SNPs were genotyped using the Sequenom MassARRAY iPLEX platform (Sequenom Inc., CA). The information regarding the primers is shown in Supplementary Table S6. The genotyping assays were performed without knowledge of the subjects' case and control status. Two blank controls (water) in each 384-well plate were used for quality control, and more than $10 \%$ of the samples were randomly selected to repeat, yielding a $100 \%$ concordance. The success rates of genotyping for these SNPs were all above $98.5 \%$. 


\section{Statistical analyses}

Differences of selected characteristics and genotype frequencies of SNPs between the GDM cases and controls were calculated using Student's $t$-test (for continuous variables) and $\chi^{2}$ test (for categorical variables). Both univariate and multivariate logistic regression analyses were used to estimate the associations between the genotypes and GDM risk by computing odds ratios (OR) and their 95\% confidence intervals (CIs). The CochranArmitage test was used for trend analyses. The $\chi^{2}$-based Q test was used to assess the heterogeneity of associations among subgroups. Haploview was employed to analyse LD parameters ( $\mathrm{r}^{2}$ and D'). PHASE software (v2.1) was used to estimate the haplotype frequencies based on the observed genotypes. All the statistical analyses were performed with SAS 9.1.3 software (SAS Institute, Cary, NC), and $P<0.05$ in a two-sided test was considered statistically significant.

\section{ACKNOWLEDGMENTS}

This work was supported in part by the National Natural Science Foundation of China (81401232, 81600685), Nanjing Medical Science and technique Development Foundation (QRX11222), the Natural Science Foundation of Jiangsu Province (BK20160141), and the Science and Technology Development Fund of the Nanjing Medical University (2015NJMUZD062).

\section{CONFLICTS OF INTEREST}

The authors have declared that no competing interests exist.

\section{Authors contributions}

XR W and J W designed the study. TC, J X, GQ L, HL, MJ C, YF Q and W W collected the data. TC and JW performed the statistical analysis and interpretation. TC wrote the report. XR W and JW revised the report. All authors reviewed the report and approved the final version.

\section{REFERENCES}

1. Reece EA, Leguizamon G, Wiznitzer A. Gestational diabetes: the need for a common ground. Lancet 2009; 373: 1789-1797.

2. Bener A, Saleh NM, Al-Hamaq A. Prevalence of gestational diabetes and associated maternal and neonatal complications in a fast-developing community: global comparisons. Int J Womens Health 2011; 3: 367-373.

3. Bellamy L, Casas JP, Hingorani AD, Williams D. Type 2 diabetes mellitus after gestational diabetes: a systematic review and meta-analysis. Lancet 2009; 373: 1773-1779.
4. Chen L, Magliano DJ, Zimmet PZ. The worldwide epidemiology of type 2 diabetes mellitus--present and future perspectives. Nat Rev Endocrinol 2012; 8: 228-236.

5. McLellan JA, Barrow BA, Levy JC, Hammersley MS, Hattersley AT, Gillmer MD, Turner RC. Prevalence of diabetes mellitus and impaired glucose tolerance in parents of women with gestational diabetes. Diabetologia 1995; 38 : 693-698.

6. Kwak SH, Kim SH, Cho YM, Go MJ, Cho YS, Choi SH, Moon MK, Jung HS, Shin HD, Kang HM, Cho NH, Lee IK, Kim SY, Han BG, Jang HC, Park KS. A genome-wide association study of gestational diabetes mellitus in Korean women. Diabetes 2012; 61: 531-541.

7. Cho YM, Kim TH, Lim S, Choi SH, Shin HD, Lee HK, Park KS, Jang HC. Type 2 diabetes-associated genetic variants discovered in the recent genome-wide association studies are related to gestational diabetes mellitus in the Korean population. Diabetologia 2009; 52: 253-261.

8. Kwak SH, Jang HC, Park KS. Finding genetic risk factors of gestational diabetes. Genomics Inform 2012; 10: 239-243.

9. Tsai FJ, Yang CF, Chen CC, Chuang LM, Lu CH, Chang CT, Wang TY, Chen RH, Shiu CF, Liu YM, Chang CC, Chen P, Chen CH, Fann CS, Chen YT, Wu JY. A genomewide association study identifies susceptibility variants for type 2 diabetes in Han Chinese. PLoS Genet 2010; 6: e1000847.

10. Below JE, Gamazon ER, Morrison JV, Konkashbaev A, Pluzhnikov A, McKeigue PM, Parra EJ, Elbein SC, Hallman DM, Nicolae DL, Bell GI, Cruz M, Cox NJ, Hanis CL. Genome-wide association and meta-analysis in populations from Starr County, Texas, and Mexico City identify type 2 diabetes susceptibility loci and enrichment for expression quantitative trait loci in top signals. Diabetologia 2011; 54: 2047-2055.

11. Chang YC, Chiu YF, Liu PH, Shih KC, Lin MW, Sheu WH, Quertermous T, Curb JD, Hsiung CA, Lee WJ, Lee PC, Chen YT, Chuang LM. Replication of genome-wide association signals of type 2 diabetes in Han Chinese in a prospective cohort. Clin Endocrinol (Oxf) 2012; 76: 365-372.

12. Chen YT, Lin WD, Liao WL, Lin YJ, Chang JG, Tsai FJ. PTPRD silencing by DNA hypermethylation decreases insulin receptor signaling and leads to type 2 diabetes. Oncotarget 2015; 6: 12997-13005. doi: 10.18632/oncotarget.4092.

13. Chagnon MJ, Uetani N, Tremblay ML. Functional significance of the LAR receptor protein tyrosine phosphatase family in development and diseases. Biochem Cell Biol 2004; 82: 664-675.

14. Pulido R, Serra-Pages C, Tang M, Streuli M. The LAR/ PTP delta/PTP sigma subfamily of transmembrane proteintyrosine-phosphatases: multiple human LAR, PTP delta, and PTP sigma isoforms are expressed in a tissue-specific manner and associate with the LAR-interacting protein LIP.1. Proc Natl Acad Sci U S A 1995; 92: 11686-11690. 
15. Veeriah S, Brennan C, Meng S, Singh B, Fagin JA, Solit DB, Paty PB, Rohle D, Vivanco I, Chmielecki J, Pao W, Ladanyi M, Gerald WL, Liau L, Cloughesy TC, Mischel PS, Sander C, Taylor B, Schultz N, Major J, Heguy A, Fang F, Mellinghoff IK, Chan TA. The tyrosine phosphatase PTPRD is a tumor suppressor that is frequently inactivated and mutated in glioblastoma and other human cancers. Proc Natl Acad Sci U S A 2009; 106: 9435-9440.

16. Ortiz B, Fabius AW, Wu WH, Pedraza A, Brennan CW, Schultz N, Pitter KL, Bromberg JF, Huse JT, Holland EC, Chan TA. Loss of the tyrosine phosphatase PTPRD leads to aberrant STAT3 activation and promotes gliomagenesis. Proc Natl Acad Sci U S A 2014; 111: 8149-8154.

17. Uetani N, Kato K, Ogura H, Mizuno K, Kawano K, Mikoshiba K, Yakura H, Asano M, Iwakura Y. Impaired learning with enhanced hippocampal long-term potentiation in PTPdelta-deficient mice. EMBO J 2000; 19: 2775-2785.

18. Zabolotny JM, Kim YB, Peroni OD, Kim JK, Pani MA, Boss O, Klaman LD, Kamatkar S, Shulman GI, Kahn BB, Neel BG. Overexpression of the LAR (leukocyte antigenrelated) protein-tyrosine phosphatase in muscle causes insulin resistance. Proc Natl Acad Sci U S A 2001; 98: 5187-5192.

19. Chagnon MJ, Elchebly M, Uetani N, Dombrowski L, Cheng A, Mooney RA, Marette A, Tremblay ML. Altered glucose homeostasis in mice lacking the receptor protein tyrosine phosphatase sigma. Can J Physiol Pharmacol 2006; 84: 755-763.

20. Zhang C, Ning Y. Effect of dietary and lifestyle factors on the risk of gestational diabetes: review of epidemiologic evidence. Am J Clin Nutr 2011; 94: 1975S-1979S.

21. Reyes-Munoz E, Castellanos-Barroso G, Ramirez-Eugenio BY, Ortega-Gonzalez C, Parra A, Castillo-Mora A, De la Jara-Diaz JF. The risk of gestational diabetes mellitus among Mexican women with a history of infertility and polycystic ovary syndrome. Fertil Steril 2012; 97: 1467-1471.

22. Hoffman L, Nolan C, Wilson JD, Oats JJ, Simmons D. Gestational diabetes mellitus--management guidelines. The Australasian Diabetes in Pregnancy Society. Med J Aust 1998; 169: 93-97. 